\section{Friedemann Garvelmann}

\title{
Traditionelle Europäische Naturheilkunde - ein eigenständiges Medizinsystem
}

\begin{abstract}
«Naturheilkunde ist Heilkunst nach den Gesetzen und mit den Mitteln der Natur Sie definiert sich weniger durch ihre $\mathrm{Me}$ thoden als durch die zugrunde liegende Denkweise.» (Friedemann Garvelmann)
\end{abstract}

Basierend auf diesem Grundsatz verstehen alle traditionellen Medizinsysteme den Menschen als untrennbaren Teil der Gesamtnatur. Das bedeutet einerseits, dass alle stofflichen Elemente der Natur im menschlichen Organismus präsent sind. In logischer Konsequenz heisst das aber auch, dass die Gesetze natürlicher Funktions- und Regulationsprozesse als energetisch-informative Prinzipien für ihn und in ihm Gültigkeit haben. Dies ist auch eine der essenziellen Grundlagen der Traditionellen Europäischen Naturheilkunde (TEN), die derzeit im deutschsprachigen Raum eine Renaissance erlebt.

Hufeland, der grosse naturheilkundliche Arzt des frühen 19. Jahrhunderts, sah die Organe des Körpers als «Werkzeuge der Lebenskraft», die dazu dienen, ihre spezifischen Funktionen für den Gesamtorganismus zu erfüllen. Diese Erkenntnis stellt die Bedeutung der Funktionalität der Organe und Gewebe in den Vordergrund - nicht deren stofflichen (histologischen) Zustand. Darin liegt auch einer der entscheidenden Unterschiede zwischen der TEN und der heutigen Schulmedizin: Das Denkund Arbeitsmodell der TEN ist funktionsorientiert, während die Schulmedizin strukturorientiert arbeitet. Hieraus folgt, dass nicht nur das grundsätzliche Verständnis von Gesundheit und Krankheit sehr unterschiedlich ist, sondern dass auch die
Diagnostik kompatibel zum angewendeten Medizinsystem erfolgen muss. Damit wird offensichtlich, dass eine schulmedizinische Diagnose (repräsentiert durch einen Krankheitsnamen) keine Basis für ein naturheilkundliches Therapiekonzept sein kann - und umgekehrt.

Die Grundlage der TEN ist die hippokratische bzw. galenistische Heilkunde, die in ständigem Wandel über 2000 Jahre lang Basis der gesamten Medizin war und erst Mitte des 19. Jahrhunderts durch die Virchow'sche Zellularpathologie ersetzt wurde. Genau zu diesem Zeitpunkt erfolgte die Unterscheidung zwischen der universitär gelehrten «Schulmedizin» und dem heterogenen Spektrum der "Alternativmedizin», zu dem auch die TEN gehört. Damit versteht sich die heutige TEN nicht als historisch überholt, sondern als sich lebendig weiterentwickelndes Medizinsystem - in Kongruenz zu seinen wissenschaftlichen und philosophischen Wurzeln. Abbildung 1 stellt das Denk- und Ar-

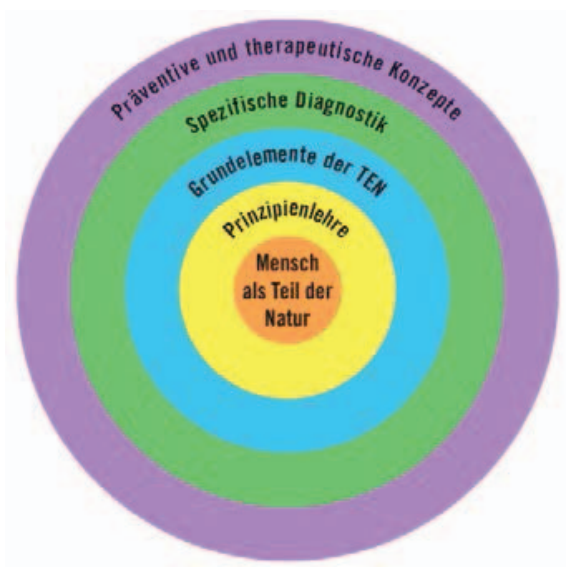

Abb. 1. Das Denk- und Arbeitsmodell der TEN. beitsmodell der TEN in Form konzentrischer Schichten oder Ebenen dar, in deren Zentrum der Mensch als Teil der Natur steht.

In der darauf folgenden Prinzipienlehre werden die natürlichen Gesetzmässigkeiten erfasst, die den physiologischen bzw. pathophysiologischen Vorgängen im Menschen zugrunde liegen. Die Prinzipienlehre ist damit das charakterisierende und strukturierende Fundament der TEN. Man unterscheidet zwischen qualitativen Prinzipien (z.B. energetisches/ stoffliches Prinzip, hyperkinetisches/ hypokinetisches Prinzip, Wärme-/ Kälteprinzip, Sulphur-/Mercurius-/ Sal-Prinzip, humorale Prinzipien usw.) und quantitativen Prinzipien (z.B. Dualität, 4 Elemente, 7 Wochentage, 12 Stunden/Monate usw.). Die Prinzipienlehre ist auch die Basis aller weiteren Schichten des Modells, die in Abbildung 2 weiter präzisiert werden.

Die im Folgenden beschriebenen Grundelemente der TEN beinhalten verschiedene Denk- und Arbeitsmodelle, die wiederum Basis sowohl der Diagnostik als auch der Therapie bzw. Prophylaxe sind.

Elementenlehre: Die eingangs erwähnten Elemente Luft, Wasser, Feuer und Erde repräsentieren die Elementarqualitäten «Wärme» und «Feuchtigkeit» bzw. deren Polaritäten mit definierten Zuordnungen:

- Luft: warm und feucht;

- Wasser: kalt und feucht;

- Feuer: warm und trocken;

- Erde: kalt und trocken.

Die Elemente wiederum werden im menschlichen Organismus durch die vier Kardinalsäfte der Humoralmedizin repräsentiert:

\section{KARGER}

Fax +4976145207 14 (c) 2013 S. Karger GmbH, Freiburg
Friedemann Garvelmann

Heilpraktiker

Hauptstrasse 8, 79790 Küssaberg-Kadelburg, Deutschland

f.garvelmann@rhizoma.de 


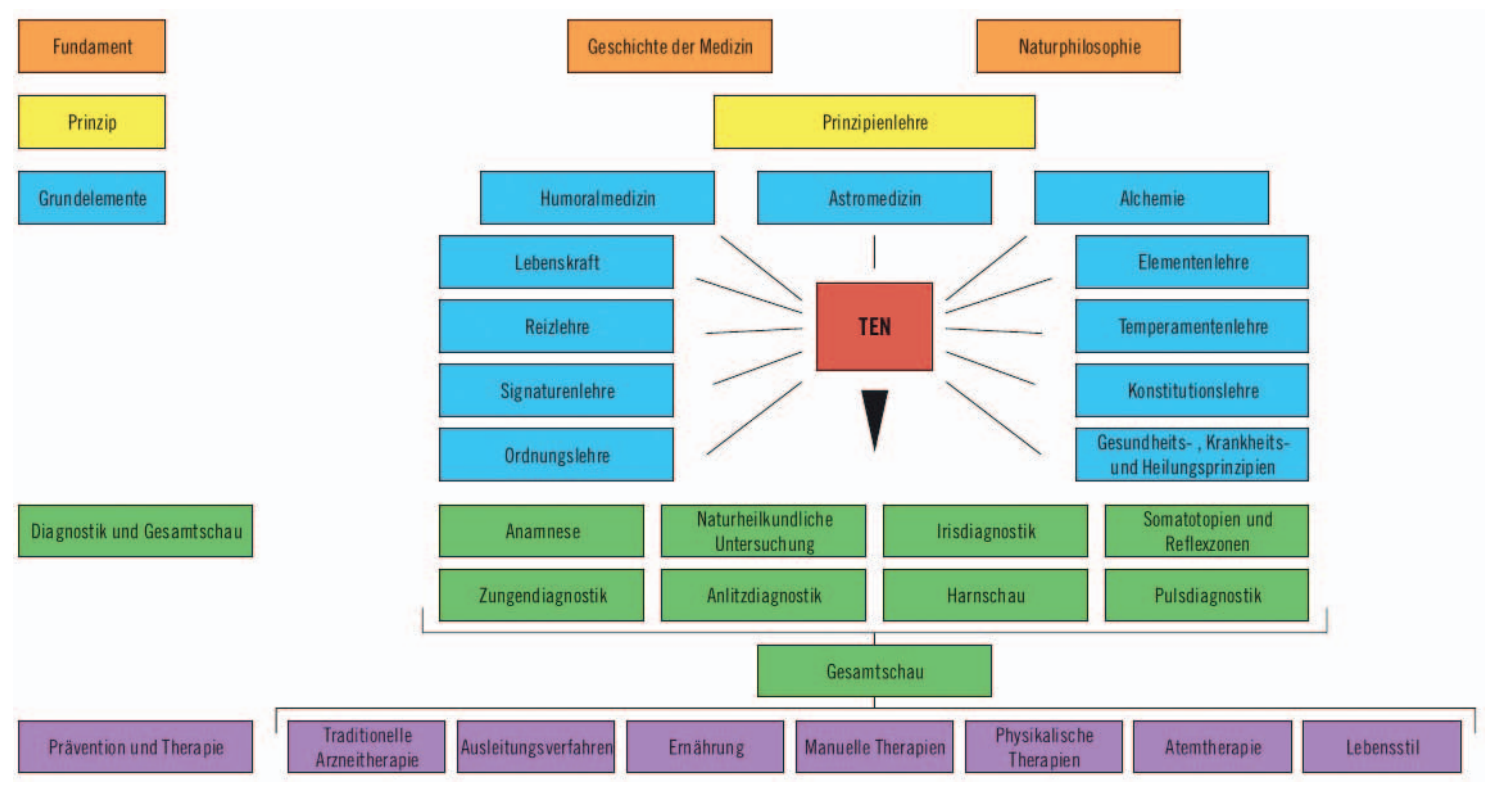

Abb. 2.

Schema der TEN.

- Sanguis (Blut): warm und feucht;

- Phlegma (Schleim): kalt und feucht;

- Cholera (gelbe Galle): warm und trocken;

- Melancholera (schwarze Galle): kalt und trocken.

Dabei dürfen die Kardinalsäfte keinesfalls als physische Körperflüssigkeiten gesehen werden, sondern als spezifische Wirkprinzipien entsprechend ihren Qualitäten.

Die Humorallehre ist Basis der humoralen Pathophysiologie, die wiederum Grundlage der humoralen Diagnostik und schlussendlich humoraler Therapiekonzepte ist. Aus der Humorallehre gehen auch die Temperamentenlehre und die gesamte Konstitutionsmedizin hervor.

Viele Bereiche, die in Abbildung 2 in einzelnen Kästchen genannt werden, sind eigentlich Teilaspekte der Elementenlehre bzw. Humoralmedizin. Damit wird auch deutlich, dass die Humoralmedizin ein unverzichtbares und charakterisierendes Grundelement der TEN ist.

Die Lebenskraft ist nach dem Verständnis der TEN das lebensspendende und -erhaltende Prinzip, das unter anderem für sämtliche Regulationsvorgänge, aber auch für Heilungsprozesse verantwortlich ist. Die Lebenskraft ist in ihren verschiedenen
Existenzformen zentrales Element der Physiologie und Pathophysiologie der TEN und wirkendes Prinzip jeder Therapie.

Die Astromedizin sieht in den kosmischen Prinzipien, die durch die Planeten repräsentiert werden, weitere Aspekte, die das menschliche Leben beeinflussen. Die Astromedizin ist vor allem in der Lehre des Paracelsus ein zentrales Element.

Die Alchemie ist das universelle naturphilosophische Modell, das sich mit den Wechselbeziehungen der stofflichen und energetischen Naturelemente und deren Wandlung befasst. Sie ist historischer Vorläufer der Chemie und Grundlage des Herstellungsprozesses spagyrischer Arzneimittel. Die alchemistischen Gesetzmässigkeiten gelten aber in jedem beliebigen Lebensbereich.

Die Reizlehre erklärt die Wahrnehmung und Verarbeitung der Reize aus der Um- und Innenwelt des Menschen und seine Reaktionen darauf. Veränderungen in diesen Bereichen sind Teil der Pathophysiologie der TEN sowie der Konstitutionslehre und fliessen in die Diagnostik und das Therapiekonzept ein.

Die Signaturenlehre hat in erster Linie praktische Bedeutung in der Heilpflanzenkunde. Sie ist eine Ana-
logie-/Entsprechungslehre, die von äusseren und inneren Merkmalen und Eigenschaften einer Pflanze Rückschlüsse auf ihre Heilqualitäten erlaubt. Im erweiterten Sinne können ähnliche Entsprechungen z.B. auch beim Menschen, bei Landschaften und bei Bergen abgeleitet werden. Auch die Physiognomik ist eine Signaturenlehre am Menschen.

Die Ordnungslehre befasst sich mit der krankheitsrelevanten bzw. salutogenetischen Bedeutung der Lebensführung («Diaita») eines Menschen. Wichtig hierbei sind: Ernährung, Lebensrhythmen, Bewegung, Sexualität, Ausscheidungen, Hygiene usw. Damit ist die Diätetik ein wichtiger Aspekt in jedem Therapiekonzept.

\section{Gesundheits-, Krankheits- und}

Heilungsprinzipien

Die TEN sieht Krankheit nicht pauschal als «unerwünschtes Ereignis», das es so schnell wie möglich $\mathrm{zu}$ beseitigen gilt, sondern analysiert sehr differenziert die Hintergründe und den biologischen Sinn eines Krankheitsprozesses. So können eine Krankheit bzw. ihre Symptome das Ergebnis folgender Kriterien sein:

- Krankheiten, deren Symptomatik aus einer biologisch sinnvollen Heilungsreaktion entsteht; Beispie- 
le: akute Entzündungen auf Oberflächenorganen (Haut, Schleimhäute), kompensatorische Eliminationsprozesse (Katarrh, Ekzem).

- Krankheiten, deren Symptomatik aus einer biologisch wider- bzw. unsinnigen Reaktion des Organismus auf die pathogenen Reize entsteht; Beispiele: allergische und autoaggressive Krankheiten, chronische Entzündungen, Rheumatosen, spasmophile Diathese, Gefässdysregulationen, Neurosen, Psychosen.

- Krankheiten, deren Symptomatik aus den organisch manifesten Folgen versagender Heilungsreaktion bzw. sonstiger erworbener und angeborener Organpathologie entsteht; Beispiele: Organinsuffizienz, Degeneration, Fehlbildungen, Verletzungen.

Die Analyse und Einordnung der Krankheit eines Patienten nach diesen Gesichtspunkten ist Teil der Diagnostik und muss im Therapiekonzept Berücksichtigung finden, um Unterdrückungsmechanismen und deren Folgen zu vermeiden.

Die nächste Ebene der spezifischen Diagnostik umfasst einige Methoden, die auch in anderen Medizinsystemen üblich sind. In der TEN werden sie aber nach deren spezifischen Kriterien modifiziert, was sowohl die Durchführung als auch die Interpretation betrifft.

Ziel der Diagnostik ist nicht die Benennung der Krankheit, sondern die Analyse der individuellen Pathophysiologie und Pathogenese - vor allem nach den Kriterien der Prinzipien- und Humorallehre.

Anamnese und körperliche Untersuchung werden so praktiziert, dass die TEN-spezifischen Prinzipien der Gesamtsituation erfasst werden.

Die körperliche Untersuchung ergibt bereits häufig Befunde, die dem Bereich Somatotopien und Reflexzonen zuzuordnen sind und pathophysiologische Wechselbeziehungen zwischen der Körperoberfläche und den inneren Organen offenbaren.
Schwerpunkt der Iris- bzw. Augendiagnostik ist die Erkennung der konstitutionellen Situation als Basis der Pathophysiologie vor allem chronischer Krankheiten. Das zugrunde liegende Denk- und Arbeitsmodell ist die Humoralmedizin, weshalb sich dieses noch relativ junge Verfahren als Weiterentwicklung nahtlos in die TEN integrieren lässt.

Die Zungendiagnostik liefert aus der Form, dem Zustand, der Farbe und dem Belag der Zunge weitere Informationen über die humorale Situation des Betroffenen.

Im Rahmen der Pulsdiagnostik wird nicht nur die Herzfrequenz gemessen, sondern vor allem die Qualität der Pulswelle (Länge, Breite, Dynamik, Unterdrückbarkeit usw.). Sie ist die wichtigste Diagnosemethode, die Informationen über die aktuelle Krankheitssituation im Sinne der Humoralmedizin gibt. Da die Pulsqualität sich kurzfristig verändern kann, lassen sich damit auch Aussagen über den Krankheitsverlauf bzw. das Ansprechen der Therapie machen.

Die Harnschau geht über die heutige quantitative Harnanalyse hinaus, indem sie beispielsweise die Farbe und Dichte, Schleimansammlungen sowie Veränderungen bei der Abkühlung des Urins im Sinne der Humoralmedizin interpretiert. Als moderne Erweiterung der TEN wird heute auch die Urin-Kochprobe verwendet.

Sämtliche Informationen der verschiedenen diagnostischen Verfahren werden zu einer diagnostischen $\mathrm{Ge}$ samtschau verknüpft, die zur Grundlage des Therapiekonzepts wird. Aus der Gesamtschau ergibt sich die prinzipielle therapeutische Zielsetzung und erst daraus die Entscheidung, mit welchen Therapieverfahren oder Kombinationen von Methoden das therapeutische Ziel optimal erreicht werden kann. Die Methoden als solche stehen also weder bei der Diagnostik noch in der Therapie im Vordergrund. Entscheidend ist deren optimale Auswahl entsprechend der individuellen Patientensituation.
Die in der TEN verwendeten Therapiemethoden (Abb. 2) dürfen als bekannt vorausgesetzt werden, weil viele hiervon auch in anderen Richtungen der Alternativmedizin angewendet werden. Darin liegt aber auch ein nicht zu unterschätzendes Problem: Die Methoden werden heute meist indikationsbezogen auf der Basis des schulmedizinischen Modells angewendet und nicht entsprechend des naturheilkundlichen Denk- und Arbeitsmodells, aus dem sie ursprünglich entstanden sind. Darunter aber leidet zwangsläufig ihre therapeutische Effizienz.

Die Grenze zwischen Naturheilkunde und «Schulmedizin mit biologischen Mitteln» liegt häufig sogar innerhalb einer Methode. Ein bekanntes Beispiel dafür ist die Phytotherapie bzw. Heilpflanzenkunde: Setzt man Pflanzen bzw. ihre Zubereitungen wirkstofforientiert und nach Indikationen ein, ist dies eine klar schulmedizinische Vorgehensweise. Verwendet man Heilpflanzen aber gemäss ihrer humoralen Qualitäten, ihres Säftebezugs, ihrer Signatur oder ihrer konstitutionellen Wirkung (um nur einige Aspekte zu nennen), wird die Heilpflanzenkunde zur systemischen Methode innerhalb der TEN.

Ähnlich verhält es sich bei der Ernährungstherapie, die nach TENKriterien niemals schematisch sein kann, sondern sich immer an der individuellen energetischen und materiellen Gesamtsituation des Patienten orientieren muss, wobei auch den Nahrungsmitteln spezifische humorale Qualitäten zugewiesen werden.

Auch die Ab- und Ausleitungsverfahren können im Interesse einer optimalen Wirkung nicht rein indikationsbezogen angewendet werden. Grundlage ist immer die allgemeine und lokale humorale Situation, die unter anderem aus dem Zustand des zu behandelnden Hautareals erkennbar ist.

Für einige der genannten Fachbereiche existiert teilweise hervorragende Literatur, die das beschriebene 
Verfahren aber meist isoliert, d.h. herausgelöst aus dem Kontext des naturheilkundlichen Gesamtsystems, behandeln.

Bisher gab es keine aktuelle Literatur, die die TEN als systematisches Ganzes mit seinem charakteristischen Denk- und Arbeitsmodell und den dazu kompatiblen diagnostischen und therapeutischen Methoden darstellte. Diese literarische Lücke wurde mit dem im Bacopa-Verlag erschienenen Buch «Grundlagen der Traditionellen Europäischen Naturheilkunde» des schweizerisch-deutschen Autorenquintetts Christian Raimann, Chrischta Ganz, Friedemann Garvelmann, Heide-Dore Bertschi-Stahl und Rosmarie Fehr-Streule geschlossen (Anmerk. d. Red.: siehe auch Buchrezension im letzten Heft). In diesem Werk beschränken sich die AutorInnen nicht auf die dogmatische Wiedergabe «alter» Quellen, sondern führen nach heutigem Wissen notwendige Adaptionen durch, ergänzen die TEN mit «modernen» Weiterentwicklungen und untermauern ihre Lehre mit vielen praktischen Beispielen und Fallbeschreibungen.

Dieses Buch legt den Grundstein für die Definition der TEN als Fachrichtung innerhalb der Alternativmedizin und ist gleichzeitig ein wertvolles Lehrbuch für die Ausund Weiterbildung von TEN-TherapeutInnen aus der Feder erfahrener (Natur-)Heilpraktiker/innen, die seit Jahrzehnten in ihren Praxen mit der TEN arbeiten und diese als DozentInnen auch lehren. Nähere Informationen: www.ten-buch.com. 